\title{
Smart Intruder Detection System with OpenCV and Python
}

\author{
P. Bhanu Teja \\ Sreenidhi Inst. Of Science and \\ Technology \\ Hyderabad, Telangana
}

\author{
P. Sandeep Kumar \\ Sreenidhi Inst. Of Science and \\ Technology \\ Hyderabad, Telangana
}

\author{
N. Dileep Reddy \\ Sreenidhi Inst. Of Science and \\ Technology \\ Hyderabad, Telangana
}

\begin{abstract}
Thefts have been on the rise in recent years. This produces a dangerous climate in which people live in terror. In today's environment, the problem of home security is a source of anxiety. The standard intruder detection systems that we currently use are extremely expensive, and there is a risk of false alerts. This issue is solved by combining OpenCV and a mobile phone to create a framework that can accurately recognize a interloper while filtrating the movements caused by objects which are moving. If an interloper is identified, the system sends a message to the user via an API called Twilio, and the footage is saved to the local storage.
\end{abstract}

Keywords: OpenCV, Twilio API, Python, IP camera, Background Subtraction.

\section{INTRODUCTION}

The presence of a human safety officer is not totally effective process of security and not totally reliable. In such scenarios, our framework provides proper interloper detection and protects the owner's property. By using this system, we can reduce robberies. So, we can react rapidly with the end goal that no damages happen in our houses. Having the interloper's face detailing, we can find him by raising a complaint against him in a police station. In the planned framework, the camera is placed in a room that can continuously capture the video. We planned our framework to undergo a client-side process whenever a gatecrasher enters the room and an alert message will be sent via Twilio API to the user or owner. Our framework is totally based on motion detection and sending an alert message using OpenCV and python.

\section{EXISTING MODEL}

In today's world, there are many existing systems for surveillance and security systems. They either need a human safety officer or need work for installing and finally which may produce false alarms. In some surveillance systems, an interloper will be detected by using video recording cameras and stores the recorded video in an external storage disk and need a huge investment for controlling it, storing, and monitoring the activities. But the footage should be examined manually by an owner to detect the gatecrasher which may lead to wastage of time as he needs to watch the entire recording to find the interloper and may miss small details while analyzing. And the second system is a radar-based system that detects an interloper by using a radar system by transmitting microwaves or radio frequency waves which will bounce off any object in its path. The other one is the intruder alarming system which includes electric alarms delegated for alerting the owner when the interloper enters. And many other systems are passive infrared motion detection systems, ultrasonic motion detection systems, vibrate sensor systems, etc.

\section{PROPOSED SYSTEM}

The proposed system uses an IP webcam application that should be installed on a mobile phone and python code for detecting the intruder and sending an alert SMS. Both will work as client-server respectively. On the client-side, the video is captured continuously and filtered. The filtered information is sent to the server-side. And it displays the status of the interloper with a time and date stamp. If the framework detects the interloper, then it starts recording the current frame and has some patience level. After the patience level drops to 0 recording process will end and the framework alerts the user or owner by sending an alert message by using a Twilio API and saves the recorded footage into the local storage.

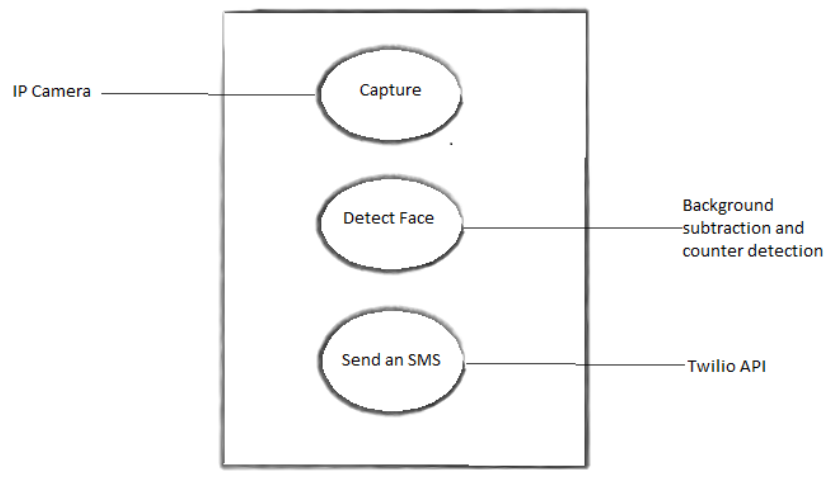

Figure. 1 System Functioning

\subsection{Proposed System Work Flow}

The system flow begins with an IP webcam capturing a live stream, and background subtraction is applied to the live streaming in order to detect the person. If the person is detected, the room occupied status will change to true; otherwise, it will be false. If the room occupied status is true, the framework begins capturing the current frame and after detecting the person, if he is not detected for certain frames, the framework waits 7 seconds before deciding whether to save the video to local storage and sends an alert message to the user, otherwise the streaming was aligned for background subtraction again. 


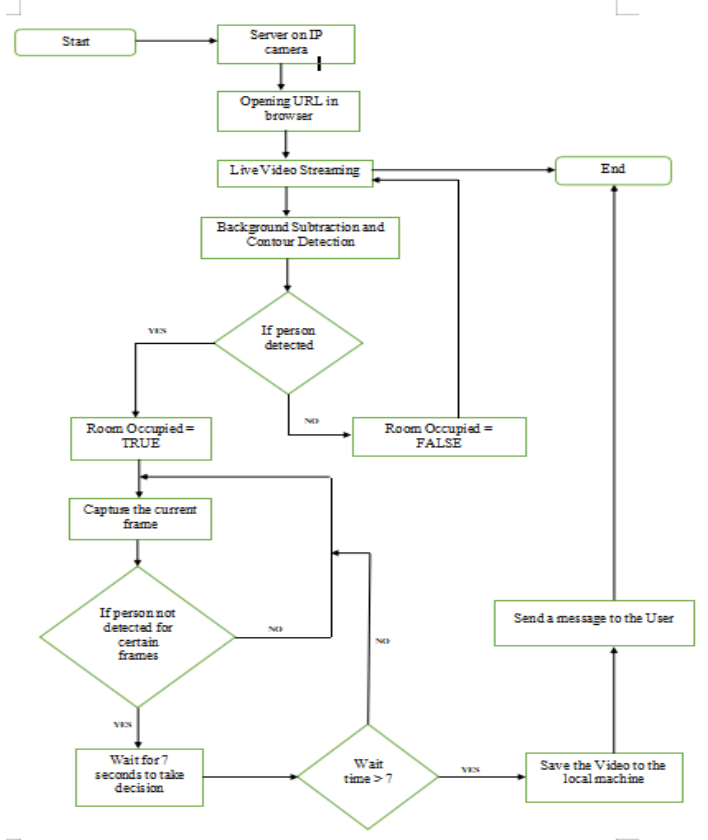

Figure.2 Flow Chart

\section{ANALYSIS}

\subsection{Software Requirements}

$\checkmark$ Operating System: Windows 10, Mac OS 11.2.2

$\checkmark$ TOOL: Jupyter Notebook

$\checkmark$ Coding Language: Python

$\checkmark$ Library: OpenCV, NumPy, Time, datetime, collections, Twilio

\subsubsection{OpenCV}

Computer vision is a space of computer programming which bases on making PC's fit for interpreting pictures. It got out during the 70's when Martin Minsky asked is student to interface a computer to a camera and getting the PC to evaluate what it saw [4]. During this time the state of art of computer vision transformed into reality. Today $\mathrm{CV}$ is consistently used with AI which setup to recognize certain features or things like remembering the portion of people [6].

\subsubsection{Python}

Python is a globally reputed language which is proposed to be significantly coherent. It is an interpreted language which resembles PERL and PHP. It is very interactive in nature and has user friendly interface. It is set to be object oriented which maintains procedure of programming that optimizes code inside objects.

\subsubsection{Twilio API}

Twilio's APIs (Application Programming Interfaces) power its foundation for correspondences. Behind these APIs is a product layer associating and upgrading interchanges networks all throughout the planet to permit your clients to call and message anybody, universally. Twilio, for instance, gives many separate REST APIs to sending instant messages, settling on telephone decisions, looking into telephone numbers, dealing with your records, and significantly more. In Twilio's environment, every item is its own API, however you will work with every one of them in generally a similar manner, regardless of whether over HTTP or utilizing
Twilio's partner libraries for a few diverse programming dialects.

\author{
4.2 Hardware Requirements \\ System: Intel $1.90 \mathrm{GHz}$ \\ $\checkmark$ Hard Disk: 1TB \\ $\checkmark$ RAM: 8 GB \\ $\checkmark$ A Mobile Phone: RAM: 2GB \\ $\checkmark$ WIFI support \\ $\checkmark \quad 5$ MP Camera \\ $\checkmark$ Storage Space: 4GB

\subsection{Application Requirements $\checkmark$ IP Camera \\ $\checkmark$ Twilio}

\section{ALGORITHMS USED}

\subsection{Background Subtraction}

The most popular Background subtraction algorithms are:

1. BackgroundSubtractorMOG() algorithm: It is one of the background subtraction algorithm which uses the concept of gaussian mixture.

2. BackgroundSubtractorMOG2() algorithm: It is same concept as BackgroundSubtractorMOG() but major difference it can provide stability even in high light intensity conditions and also stability while identifying the shadows in the each frames.

3. Geometric multigrid: It makes uses of statistical method and per pixel Bayesian segmentation algorithm .

The process becomes more complicated when there is a shadow without the actual image or appearance of the object. Simple background subtraction algorithms will consider the moving or still shadows as foreground which reduces the accuracy of the system. The solution for above complications will be solved openCV.js which can simple and easy to use. The below constructor is the solution.

The cv2.createBackgroundSubtractorMOG2() takes in 3 contentions:

1. detectsSadows: Now this calculation can likewise recognize shadows, on the off chance that we pass in detectShadows=True argument in the constructor. The capacity to identify and dispose of shadows will give us smooth and powerful outcomes. Empowering shadow discovery somewhat diminishes speed.

2. history: This is the quantity of edges that is utilized to make the foundation model, increment this number if your objective item frequently stops or stops briefly.

3. varThreshold: This edge will help you sift through commotion present in the casing, increment this number if there are heaps of white spots in the edge. In spite of the fact that we will likewise utilize 
morphological activities like disintegration to dispose of the commotion

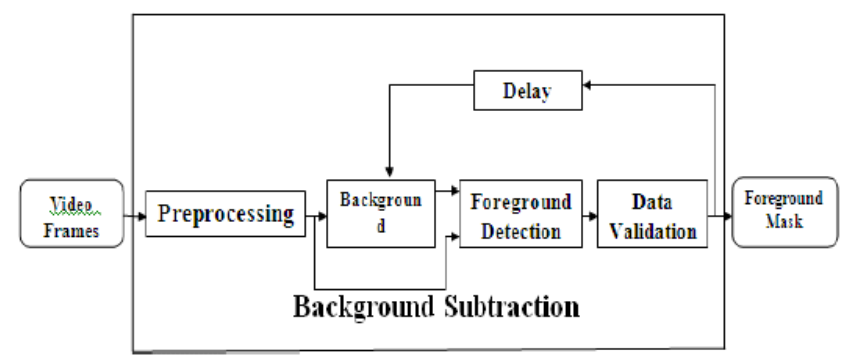

Figure. 3 Background Subtraction Flow Chart

\section{IMPLEMENTATION}

We will be splitting the project into four parts which have their own functionality.

1. Accessing the Live stream from your telephone to OpenCV.

2. How to utilize the Twilio API to send an Alert SMS.

3. Building a Motion Detector with Background Subtraction and Contour recognition.

4. Making the Final Application.

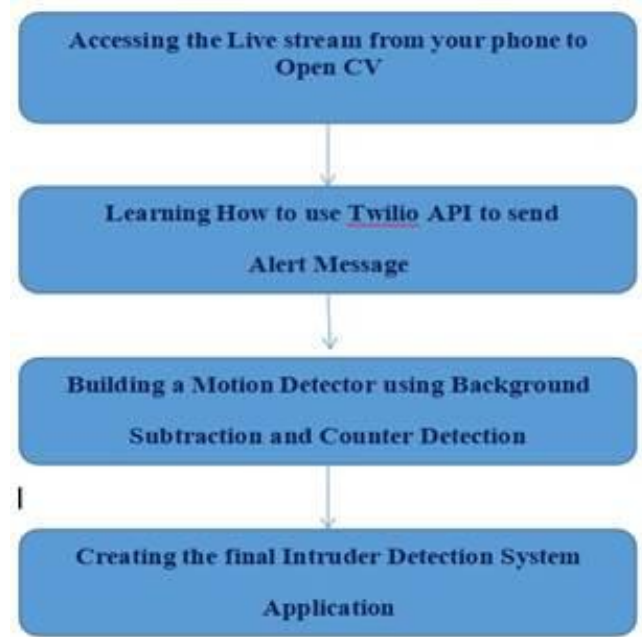

Figure. 4 Background Subtraction Flow Chart

In this framework, we developed an intruder detection system in which users can use an Ip camera application to detect an intruder. Here, live streaming is captured through the cv2.VideoCapture() method by copying the IP address of an IP camera. A person can be detected by applying a background subtraction algorithm to the video being captured. And Twilio library is installed to write a code for sending alert message whenever a person is detected by the framework. Lastly, a final application is created by blending all steps involved in the framework.

\section{RESULT}

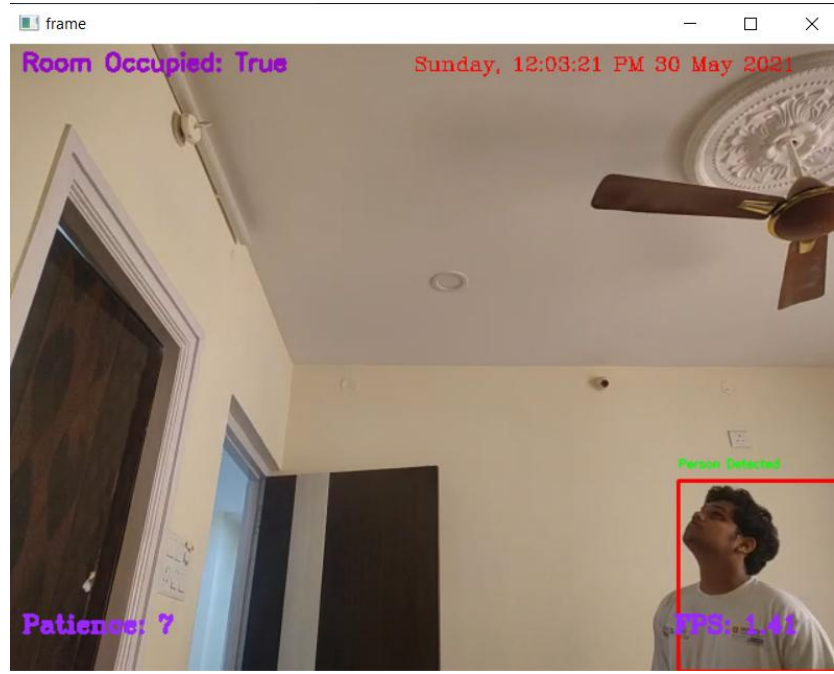

Figure. 5 Room occupied is true

Here we can see that the Room Status is TRUE as the person is detected in the frame. The time in the specified format is given on the top right-hand side of the frame is taken down. The contours drawn are of red colour of the accurate visibility to the user. The FPS meter and the Patience level are being displayed on the bottom side right and left side to the frame respectively.

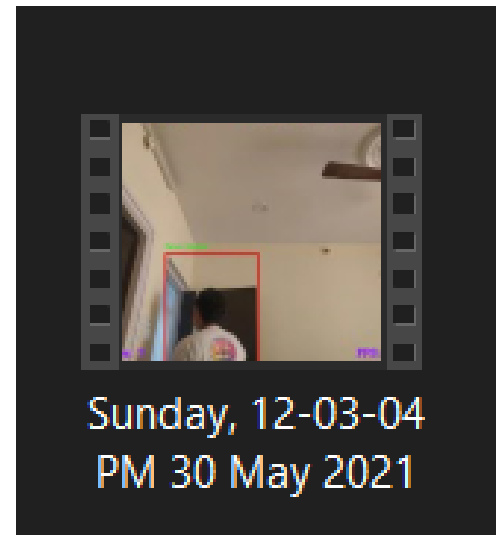

Figure. 6 Saved video of intruder with time stamp 


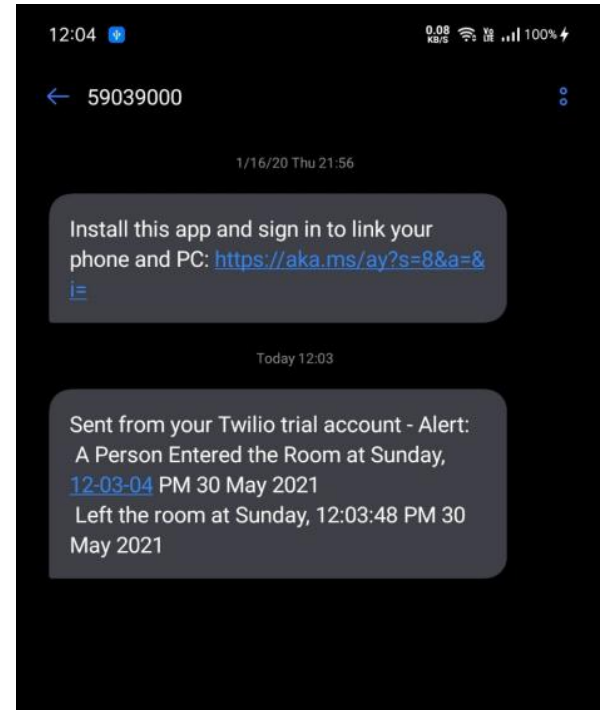

Figure. 7 Alert Message send by Twilio API

\section{CONCLUSION}

The application presented in this project is a proof of concept on how to create an application that can detect human motion in a room, which also saves video clips to the local machine. The created application is adapted to a smart phone with a 5 mega pixel camera to yield optimal functionality. The application also needs an internet connection and an IP camera application from the Google Play Store. The created application yields a good detection result with greater accuracy on labeling the acquired video clips with a proper file name (Date and Time Stamp) i.e., labeling the image if it contains people or not. The gathering of SMS security cautions which has sent from security gadget through SMS sending API when a interloper has been recognized. The application is suitable in an environment which is static and the movement that occurs is caused by human movement, for example a conference room. The application's motion detection has its limits, for example the application cannot tell the difference between moving objects and human movement.

The system is made up of both hardware and software components that work together to provide an effective motion detecting method. The background deduction strategy was applied in OpenCV execution and outcome got from the directed the analyses recommended the better-precision of an interloper-based movement identification system, taking out bogus alarms. The suggested system is partially self-contained and wireless, resulting in a security system that is dependable, resilient, simple to use, and inexpensive. WIFI has been used to connect to the internet for communication. Various procedures have been successfully tried, and the findings have been reported, I conclude.

\section{FUTURE ENHANCEMENT}

This system has a wide range of applications in a variety of sectors, including banking, forensics, and so on. The reason this system is so beneficial is that it is extremely small and enables facial detection as well as immediate email notification. In addition, face recognition may be used in the future. Any security system's most important component is recognition. Typically, for the greatest recognition system, we need a well-trained database that can serve as the foundation for our recognition. To get the database, first gather the subject's photographs. Person for the acknowledgment We will be able to give facial recognition once we have acquired and trained our system.

For face identification, we can employ the local binary pattern histogram (LBPH). This strategy aids in the creation of a recognition model. The picture is then transformed to grayscale. The picture pixels are then compared in a clockwise or anti-clock-wise way to their neighbor's. For each image, a histogram is created, normalization is performed, and a feature vector is constructed. These feature vectors may now be used to categorize photos and identify the texture using specific techniques. After the face has been recognized, it is reviewed to determine whether it is a recognizable face. As a result, we combine face detection and recognition to create a smart monitoring system for domestic use in our daily lives.

It could be a good idea to make the binary/movement thresholds dynamic and dependent on the average distance in the room in order to make the programmed operate better in rooms of varying sizes. Because if a person goes far away from the camera, the visual difference will be smaller than if the person moved closer to the camera.

\section{ACKNOWLEDGMENTS}

We express our heartiest thanks to Management of Sreenidhi Institute of Science and Technology, for providing the necessary facilities to undergo this project. We would like to extend our heartiest thanks to the Mrs. Shailaja Mantha, Associate Professor, Department of Electronics \& Computer Engineering whose valuable supervision and guidance in making out of this project successfully. We would like to thank our project coordinator Mrs. K. Naga Sailaja, Assistant Professor, Department of Electronics \& Computer Engineering who has been an excellent guide and also a great source of inspiration to our work. We would like to extend our heartiest thanks to the Dr. D. Mohan, Professor, HOD of ECM, whose valuable supervision in making out of this project successful.

\section{REFERENCES}

[1] M. Piccardi (October 2004). "Background subtraction techniques: a review". IEEE International Conference on Systems, Man and Cybernetics 4. pp. 30993104.doi:10.1109/icsmc.2004.1400815

[2] Yoshida, T., 2004. Background differencing technique for image segmentation based on the status of reference pixels." International Conference on Image Processing ICIP'04, pages 3487-3490, Singapore, October 24-27, 2004

[3] Elhabian, S., K. El-Sayed and S. Ahmed, 2008 Moving object detection in spatial domain using background removal techniques - State-of-Art. Recent Pat on Computer Sci., 1(1): 32-54

[4] Papert, The summer vision project, Massachusetts Institute of technology, 1996

[5] Efstration Graves, A brief history of computer vision [Internet]. 2016 [updated 05/05- 2016, cited 05/05-2016]. Available at: http://www.egavves.com/a-brief-historyofcomputer-vision/\#sthash.b1 miJmj3.dpbs

[6] Roqueiro, Petrushin, Counting people using video cameras, Department of Computer Science, University of Illinois at Chicago, Chicago, IL 60607, USA, 2006 
International Journal of Computer Applications Technology and Research

Volume 10-Issue 06, 178-182, 2021, ISSN:-2319-8656

[7] Havaei1, Jodoin1, Efficient interactive brain tumour segmentation as within-brain $\mathrm{kNN}$ classification. University Sherbrooke, Canada. 2014

[8] Mobileye, Mobileye - Our Vision. 2016 [updated 04/252016, cited 25/04-2016]. Available at: http://www.mobileye.com/

[9] Vargas, M., F. Barrero and J.M. Milla, 2008. An Enhanced Background Estimation Algorithm for Vehicle
Detection in Urban Traffic Video Proceedings of the 11 International IEEE Conference on Intelligent

Transportation Systems, Beijing, China, October 12-15, 2008.

[10] Shanker, Lai, Android porting concepts, Electronics Computer Technology (ICECT), 2011 3rd International Conference on, Kanyakumari, 2011, pp. 129-133. 